\title{
Pair Function for the Rectangular Ising Ferromagnet
}

D. B. Abraham*

Department of Theoretical Chemistry, Oxford University, Oxford OX1 3TG, England, and Aspen Center for Physics, Aspen, Colorado 81611, USA

\begin{abstract}
A representation of the pair correlation function for the rectangular Ising model in zero magnetic field is derived using a new spinor technique; this enables the scaling limit to be established, as well as several analytical properties of the scaling functions.
\end{abstract}

\section{Introduction}

In this paper a representation for the pair function will be obtained using the matrix element results of the previous paper; the equations therein to which we shall refer freely, will be denoted I.n.m. In this case the matrix element $\left\langle\Phi_{-}\left|G_{\alpha_{n}} \ldots G_{\alpha_{1}} \sigma_{1}^{x}\right| \Phi_{+}\right\rangle$will be needed. They can be obtained in terms of the $\left\langle\Phi_{-}\left|G_{\alpha_{n}} \ldots G_{\alpha_{1}}\right| \Phi_{+}\right\rangle$by using the definition of $G_{\alpha}$.

By analogy with [1, Eq. (3.4)], consider the definition D.1.

$$
F_{M}^{x}\left(\left(e^{i \alpha}\right)_{n}\right)=M^{n / 2} \exp i \sum_{1}^{n}\left(\alpha_{j}+\Theta\left(\alpha_{j}\right)\right)\left\langle\Phi_{-}\left|G_{\alpha_{n}} \ldots G_{\alpha_{1}} \sigma_{1}^{x}\right| \Phi_{+}\right\rangle \text {. }
$$

Then using [1], Eq. (2.9), (2.14), and (2.28) it follows that

$$
\begin{aligned}
F_{M}^{x}\left((z)_{n}\right)= & \sum_{j=1}^{n}(-1)^{j} F_{M}\left(\Delta_{j}(z)_{n}\right)\left\{1 / \Theta\left(z_{j}\right)\right. \\
& \left.+M^{-1} \sum_{z \in S_{M}^{-}} \Theta(z) f_{M}\left(z, z_{j}\right)\right\},
\end{aligned}
$$

where the functions $F_{M}\left((z)_{n}\right)$ and $f_{M}(z, t)$ are defined in $[1$, Eq. (3.4)]. The appropriate limiting form is $F^{x}\left((z)_{n}\right)$ given by

$$
F^{x}\left((z)_{n}\right)=\sum_{j=1}^{n}(-1)^{j} F\left(\Delta_{j}(z)_{n}\right)\left\{\frac{\mathscr{P}}{2 \pi^{i}} \int_{C_{1}} \frac{d z}{z} \Theta(z) f\left(z, z_{j}\right)+1 / \Theta\left(z_{j}\right)\right\}
$$

* Supported by the Fonds National Suisse de la Recherche Scientifique, by the National Science Foundation Grant No. PHY76-17191, and by the National Research Council of Canada Grant No. NRC A9344 
Table 1. Data for contraction functions (1.8), factorisation of (1.6) and (1.7) and initial conditions for Pfaffian recurrence relations

\begin{tabular}{|c|c|c|c|c|}
\hline & \multicolumn{2}{|l|}{$T>T_{\mathrm{C}}$} & \multicolumn{2}{|l|}{$T<T_{\mathrm{C}}$} \\
\hline & $V$ & $V^{1}$ & $V$ & $V^{1}$ \\
\hline $\begin{array}{l}\Theta_{+}(z) \\
\Theta_{-}(z)\end{array}$ & $\begin{array}{l}{\left[\left(z-B^{-1}\right)(z-A)\right]^{1 / 2}} \\
(A / B)^{1 / 2}\left[\left(z-A^{-1}\right)(z-B)\right]^{1 / 2} / z\end{array}$ & $\begin{array}{l}{\left[(z-A) /\left(z-B^{-1}\right)\right]^{1 / 2}} \\
(A B)^{-1 / 2}\left[(z-B) /\left(z-A^{-1}\right)\right]^{1 / 2}\end{array}$ & $\begin{array}{l}{[(z-B) /(z-A)]^{1 / 2}} \\
(A / B)^{1 / 2}\left[\left(z-A^{-1}\right) /\left(z-B^{-1}\right)\right]^{1 / 2} / z\end{array}$ & $\begin{array}{l}{[(z-A)(z-B)]^{1 / 2}} \\
{[(A z-1)(B z-1)]^{-1 / 2}}\end{array}$ \\
\hline $\begin{array}{l}F(z) \\
F(\phi)\end{array}$ & $\begin{array}{l}0 \\
\hat{m} / \cosh K_{1}^{*}\end{array}$ & $\begin{array}{l}0 \\
\hat{m}\end{array}$ & $\begin{array}{l}{[(z-A) /(z-B)]^{1 / 2}} \\
0\end{array}$ & $\begin{array}{l}(A B)^{1 / 2} m^{*} / \cosh K_{1}^{*}\left[\left(z-A^{-1}\right)\left(z-B^{-1}\right)\right]^{1 / 2} \\
0\end{array}$ \\
\hline$g(z)$ & $\begin{array}{l}1 / \Theta_{+}(z) \\
\hat{m}=\left[1-\left(\sinh 2 K_{1} \sinh 2 K_{2}\right)^{2}\right]^{1 /} \\
A=\operatorname{coth} K_{1}^{*} \operatorname{coth} K_{2}\end{array}$ & & $\begin{array}{l}1 / \Theta_{+}(z) \\
m^{*}=\left[1-\left(\sinh 2 K_{1} \sinh 2 K_{2}\right)^{-2}\right]^{1 / 8} \\
B=\tanh K_{1}^{*} \operatorname{coth} K_{2}\end{array}$ & \\
\hline
\end{tabular}

with

$$
F\left((z)_{2 n}\right)=\sum_{2}^{2 n}(-1)^{j} f\left(z_{1} z_{j}\right) F\left(\Delta_{1 j}(z)_{2 n}\right)
$$

and

$$
F\left((z)_{2 n+1}\right)=\sum_{1}^{2 n+1}(-1)^{j} F\left(z_{j}\right) F\left(\Delta_{j}(z)_{2 n+1}\right) .
$$

The general solutions of (1.4) and (1.5) were developed in [1] by Wiener-Hopf techniques. For the Ising model appropriate functions $\Theta$ are

$$
\begin{gathered}
V: \Theta(z)=\left(\frac{A}{B}\right)^{1 / 2} \frac{1}{z}\left[\frac{\left(z-A^{-1}\right)(z-B)}{(z-A)\left(z-B^{-1}\right)}\right]^{1 / 2}, \\
V^{\prime}: \Theta(z)=\frac{1}{(A B)^{1 / 2}}\left[\frac{(z-A)(z-B)}{\left(z-A^{-1}\right)\left(z-B^{-1}\right)}\right]^{1 / 2} .
\end{gathered}
$$

The associated function $f(z, t)$, is of the form

$$
f(z, t)=\frac{z t}{z t-1}\left(g(z) g^{-1}\left(t^{-1}\right)-g(t) g^{-1}\left(z^{-1}\right)\right)
$$

both for $T>T_{C}$ and $T<T_{C}$ (see [1, Eqs. (4.7) and (4.16)]. The factorisations of (1.6) and (1.7) which can be constructed by inspection, are given in Table 1, together with the normalized form of $F(z)$ and the function $g(z)$ of (1.8). Equation (1.2) is now developed in the obvious limiting form by expanding the appropriate Pfaffians using the results of Table 1.

For $T>T_{C}$, one has

$$
\begin{aligned}
F^{x}\left((z)_{2 n+1}\right)= & \sum_{1}^{2 n+1}(-1)^{j} F\left(\Delta_{j}(z)_{2 n+1}\right)\left\{1 / \Theta\left(z_{j}\right)\right. \\
& \left.+\frac{\mathscr{P}}{2 \pi i} \int_{C_{1}} \frac{d z}{z} \Theta(z) f\left(z, z_{j}\right)\right\} .
\end{aligned}
$$


Using Table 1, and performing a few contour integrals gives the result

$$
F^{x}\left((z)_{2 n+1}\right)=-\Theta_{-}(\infty) \sum_{1}^{2 n+1}(-1)^{j} \Theta^{-1}\left(z_{j}\right) F\left(\Delta_{j}(z)_{2 n+1}\right),
$$

where the Pfaffian on the right hand side is given in (1.4) and in Table 1.

On the other hand, when $T<T_{C}$ more care is necessary. Using (1.5), one has

$$
\begin{aligned}
F^{x}\left((z)_{2 n}\right)= & -\frac{1}{2 \pi i} \int_{C_{1}} \frac{d z}{z} \Theta(z) F(z) F\left((z)_{2 n}\right) \\
& +\sum_{2}^{2 n+1}(-1)^{j} F\left(z_{j}\right) \sum_{k=2}^{2 n} P_{k j} F\left(\Delta_{1 k j}(z)_{2 n+1}\right)\left\{1 / \Theta\left(z_{k}\right)\right. \\
& \left.+\frac{\mathscr{P}}{2 \pi^{i}} \int_{C_{1}} \frac{d z}{z} \Theta(z) f\left(z, z_{k}\right)\right\} .
\end{aligned}
$$

The second integral in (1.11) is easily carried out using the Wiener-Hopf factorisation to give the contribution

$$
\sum_{j=2}^{2 n+1}(-1)^{j} \sum_{k=2}^{2 n} F\left(z_{k}\right) P_{k j} F\left(\Delta_{1 k j}(z)_{2 n+1}\right),
$$

where

$$
P_{k j}=(-1)^{k}\left(1-\delta_{k j}\right) \operatorname{Sgn}(j-k) .
$$

It follows immediately that this vanishes; the first integral in (1.11) is just the normalisation condition for $F(z)$. Using the notation

$$
m^{*}=\lim _{M \rightarrow \infty}\left\langle\Phi_{-}\left|\sigma_{1}^{x}\right| \Phi_{+}\right\rangle
$$

one has

$$
F^{x}\left((z)_{2 n}\right)=-m^{*} \tilde{F}\left((z)_{2 n}\right),
$$

where the boundary condition for the Pfaffian on the right hand side is

$$
F(\emptyset)=1 \text {. }
$$

Thus one may rewrite (1.14) and (1.15) as

$$
F^{x}\left((z)_{2 n}\right)=F\left((z)_{2 n}\right),
$$

where $F\left((z)_{2 n}\right)$ is given by (1.4) and Table 1, with the initial condition given there. The next section applies (1.10) and (1.16) to evaluate the pair correlation function.

\section{The Pair Function}

The pair function is given as a special case of [1].

$$
\begin{aligned}
\varrho_{2}(r)= & \lim _{M \rightarrow \infty} \sum_{0}^{\infty} \frac{1}{n ! M^{n}} \sum_{z_{1} \ldots z_{n}}\left|F_{M}^{x}\left((z)_{n}\right)\right|^{2} \\
& \cdot \exp -\sum_{1}^{n}\left(|x| \gamma\left(\omega_{j}\right)-i y \omega_{j}\right),
\end{aligned}
$$


Table 2. Edge weights for graphical representation in real form

\begin{tabular}{ll}
\hline & $e\left(\omega_{1}, \omega_{2}\right)$ for symmetrisation $V$ \\
\hline$T>T_{\mathrm{C}}$ & $\left(\sinh \gamma\left(\omega_{1}\right)-\sinh \gamma\left(\omega_{2}\right)\right) / 2 \sin \left(\omega_{1}+\omega_{2}\right) / 2$ \\
$T<T_{C}$ & $\left(p\left(\omega_{1}\right) q\left(\omega_{2}\right)-p\left(\omega_{2}\right) q\left(\omega_{1}\right)\right) / 2 \sin \left(\omega_{1}+\omega_{2}\right) / 2$ \\
& $p(\omega)=(2 \cos \omega-A-1 / A)^{1 / 2}$ \\
& $q(\omega)=(2 \cos \omega-B-1 / B)^{1 / 2}$ \\
\hline
\end{tabular}

where $\boldsymbol{r}=(x, y)$ and the sums are to be taken independently over $z$ such that $z^{M}=1$. The thermodynamic limit can now be taken using the estimates of $\left\|\delta f_{M}\right\|$ given in the last paper. The limiting form is

$$
\begin{aligned}
\varrho_{2}(\boldsymbol{r})= & \sum_{0}^{\infty} \frac{1}{n !(2 \pi)^{n}} \int \cdots \int d(\omega)_{n} \\
& \cdot\left|F^{x}\left(\left(e^{i \omega}\right)_{n}\right)\right|^{2} \exp -\sum_{1}^{n}\left(|x| \gamma\left(\omega_{j}\right)-i y \omega_{j}\right) .
\end{aligned}
$$

An instructive graphical representation can be given for the terms in the Pfaffian expansion of (2.2). This will be useful in examining the "scaling limit" of the pair function.

The definition (1.4) of the Pfaffian (see also [2]) which will be denoted $P f(f)$, where $f$ is referred to as the contraction function, is equivalent to the following [3]

$$
P f(f)=\sum_{\pi} \varepsilon(P(\pi)) e_{j_{1} j_{2}} \ldots e_{j_{2 n-1} j_{2 n}}
$$

with $e_{j k}=f\left(e^{i \omega_{j}}, e^{i \omega_{k}}\right)$ and where the sum is over all partitions $\pi$ of the integers $1, \ldots, 2 n$ in $n$ unordered pairs. Here $P(\pi)$ is the permutation of $1, \ldots, 2 n$ which is equivalent to $\pi$, and $\varepsilon(P(\pi))$ is its signature, which is unique since the contraction is antisymmetric. Note that a permutation within a pair of in (2.3) leaves $P f(f)$ unchanged, as does a rearrangement of the pairs themselves. This suggests how to handle $P f(f)^{*}$ : take given partitions $\pi$ and $\pi^{\prime}$ for $P f(f)$ and for $P f(f)^{*}$, which will be denoted

$$
\pi=j_{1} j_{2}\left|j_{3} j_{4}\right| \ldots \mid j_{2 n-1} j_{2 n}
$$

and

$$
\pi^{\prime}=j_{1}^{\prime} j_{2}^{\prime}\left|j_{3}^{\prime} j_{4}^{\prime}\right| \ldots \mid j_{2 n-1}^{\prime} j_{2 n}^{\prime} .
$$

Given $j_{2}$ is $(2.4 \mathrm{a})$ recorder $\pi^{\prime}$ so that $j_{1}^{\prime}=j_{2}$. Then $j_{2}^{\prime}$ is fixed, either equal to $j_{1}$, or else choose $j_{3}=j_{2}^{\prime}$. Since this process is finite, a stage must eventually be reached with $-j_{r}^{\prime}=j_{1}$. Choose a number in $1, \ldots, 2 n$ not already used for $j_{r+1}$ and repeat the procedure. Thus $|P f(f)|^{2}$ can be written as a union of vertex and edge disjoint cycles which cover the points $1, \ldots, 2 n$. Note that the cycles each have an even number of points. The associated permutations may be written as

$$
\begin{aligned}
P & =j_{1} j_{2} \ldots j_{r-1} j_{k} j_{r+1} \ldots j_{r+s} \ldots, \\
P^{\prime} & =j_{2} j_{3} \ldots j_{r} j_{1} j_{r+2} \ldots j_{r+1} \ldots,
\end{aligned}
$$


so that $P^{\prime}=P P^{\prime \prime}$ where $P^{\prime \prime}$ is a product of cyclic permutations. Each cycle has a permutation signature of -1 .

Returning to (1.10), the high and low temperature results must be carefully distingiushed. When $T>T_{C}$ the expansion of $\left|F^{x}\left(\left(e^{i \omega}\right)_{2 n+1}\right)\right|^{2}$ will contain a chain of contractions, alternately from $P f(f)$ and $P f(f)^{*}$ connecting the factors $g$ and $g^{*}$. For $T<T_{C}$ such open chains do not occur. The weighting of the graphs is greatly simplified by a suitable rationalisation of the factors in $f\left(e^{i \omega_{1}}, e^{i \omega_{2}}\right)$ and $g\left(e^{i \omega}\right)$ to produce the real edge and vetex weights which will be encountered in the following graphical rules:

1. Only simple graphs $G=\{V, E\}$ occur.

2. For a given vertex set $V$ with $|V|=n$ assign to each vertex an integration variable $\omega \in[0,2 \pi]$, and a vertex weight

$$
v(\omega)=G(\omega \mid \boldsymbol{r}) / 2 \pi
$$

where

$$
G(\omega \mid \boldsymbol{r})=\exp (-|x| \gamma(\omega)+i y \omega)
$$

with $\boldsymbol{r}=(x, y)$.

3. If $T<T_{\mathrm{C}},|V|$ must be even. $E$ is a union of edge and vertex disjoint cycles, each of which contains an even number of edges. A cycle given as an edge sequence

$$
\left\{\left(v_{1}, v_{2}\right)\left(v_{2}, v_{3}\right) \ldots\left(v_{2 n-1}, v_{2 n}\right)\left(v_{2 n} \sigma_{1}\right)\right\}
$$

has an edge weight

$$
E\left((\omega)_{2 n}\right)=-e\left(\omega_{2 n}, \omega_{1}\right) \prod_{1}^{2 n-1} e\left(\omega_{i}, \omega_{i+1}\right),
$$

where $\omega_{i}$ is the label of vertex $i$. The edge weights $e(\omega, v)$ are given in Table 2 . Notice that $e(\omega, v)=-e(v, \omega)$, so that the order of labelling in (2.8) must be observed.

4. If $T>T_{C}$, then $|V|$ must be odd. There will be a single open chain

$$
\left\{\left(v_{1}, v_{2}\right), \ldots,\left(v_{2 n}, v_{2 n+1}\right)\right\}
$$

with an edge weight

$$
E\left((\omega)_{2 n+1}\right)=\prod_{1}^{2 n} e\left(\omega_{i}, \omega_{i+1}\right) .
$$

The remainder of the vertices are covered by $E$ as in 3 above.

5. Sum over all allowed graphs.

The graphical results may be cast into a simple form by introducing a bounded operator $A_{\boldsymbol{r}}$ on $L^{2}([0,2 \pi])$ defined by

$$
\left(A_{\boldsymbol{r}} f\right)(\omega)=i h(\omega \mid \boldsymbol{r}) \int_{-\pi}^{\pi} d \mu(v) e(\omega, v) \cdot h(v \mid \boldsymbol{r}) f(v),
$$

where

$$
h(\omega \mid \boldsymbol{r})=(G(\omega \mid \boldsymbol{r}))^{1 / 2}
$$


and

$$
d \mu(\omega)=d \omega / 2 \pi \sinh \gamma(\omega) .
$$

Thus we have

$$
E\left((\omega)_{2 n}\right)=(-1)^{n+1} \operatorname{Tr}\left(A_{\boldsymbol{r}}^{2 n}\right)
$$

and

$$
E\left((\omega)_{2 n+1}\right)=(-1)^{n}\left(h, A_{r}^{2 n} h\right)
$$

with

$$
(f, g)=\int_{-\pi}^{\pi} f^{*}(v) g(v) d \mu(v) .
$$

Two important properties of $A_{\boldsymbol{r}}$ are expressed in

Proposition 2.1. 1. If $y=0$, then $A_{\boldsymbol{r}}$ is self-adjoint.

2. $A_{\boldsymbol{r}}$ is of Hilbert-Schmidt type whenever $x \neq 0$.

The proof of Proposition 2.1 (1) is elementary; the other assertion follows from the estimate

$$
\begin{aligned}
& \iint_{-\pi}^{\pi}\left|A_{\boldsymbol{r}}\left(\omega_{1}, \omega_{2}\right)\right|^{2} d \mu\left(\omega_{1}\right) d \mu\left(\omega_{2}\right) \\
& \leqq \sup _{\omega}\left|e\left(\omega_{1}, \omega_{2}\right)\right|^{2}\left(\int_{-\pi}^{\pi} e^{-|x| \gamma(\omega)} d \mu(\omega)\right)^{2} .
\end{aligned}
$$

The linked cluster theorem may now be used to sum over all the cycles which occur in the graphical representation in (2.2), as described above, to give, formally at least,

$$
\varrho_{2}(\boldsymbol{r})=\left\{\left(\hat{m}\left(K_{1}^{*}, K_{2}\right) / \cosh K_{1}^{*}\right) H(\boldsymbol{r}), \text { resp. } m^{*}\left(K_{1}^{*}, K_{2}\right)\right\} \exp K(\boldsymbol{r})
$$

for $\left\{T>T_{C}\right.$, resp. $\left.T<T_{C}\right\}$. In the above

$$
H(\boldsymbol{r})=\left(h,\left(1+A_{\boldsymbol{r}}^{2}\right)^{-1} h\right)
$$

and

$$
K(\boldsymbol{r})=\frac{1}{2} \operatorname{Tr} \log \left(1+A_{\boldsymbol{r}}^{2}\right) .
$$

It is known [4] that the range of convergence of results obtained by applying the linked cluster theorem may well be reduced. But the convexity of the log function gives

$$
|K(\boldsymbol{r})| \leqq \operatorname{Tr} A_{(x, 0)}^{2}
$$

so that, provided $x \neq 0$, Proposition 2.1 implies that the range of convergence (in $x$ ) of (2.16) is undiminished.

\section{The Scaling Limit}

One of the fundamental beliefs of statistical mechanics is that in the critical region only spatial dependence with distance scaled by the correlation length is impor- 
tant. Of course the above remark is tenuous since neither critical region nor correlation length is necessarily uniquely defined. In the present problem, however, $\gamma(0)$ (see [1]) plays the role of an inverse correlation length and $\gamma(0) \sim|t|$ as $t=\left(T-T_{C}\right) / T_{C} \rightarrow 0 \pm$. Here we shall investigate the limit

$$
F_{ \pm}(s)=\lim _{t \rightarrow 0 \pm}|t|^{p} \varrho_{2}(s / \gamma(0) ; t)
$$

where $p$ is a constant which will be chosen. Monotonicity, continuity and decay properties of $F_{ \pm}(s)$ will be derived.

Consider a contribution to $\varrho_{2}(r)$ due to a cycle of length $2 n$, denoted $L_{2 n}$. Taking the limit $t \rightarrow 0 \pm$ with $s=|\boldsymbol{r}| / \gamma(0)$ fixed and $\sinh \theta_{j}=\omega_{j} / \gamma(0)$ gives

$$
\begin{aligned}
L_{2 n}= & \left(\frac{1}{2 \pi}\right)^{2 n} \int_{-\infty}^{\infty} \cdots \int d(\theta)_{2 n} \prod_{1}^{2 n} \exp \left(-\left|s_{1}\right| \cosh \theta_{j}-i s_{2} \sinh \theta_{j}\right) \\
& \cdot \tanh \left(\frac{\theta_{1}-\theta_{2}}{2}\right) \prod_{1}^{2 n-1} \tanh \left(\frac{\theta_{j+1}-\theta_{j}}{2}\right) .
\end{aligned}
$$

Setting $\left|s_{1}\right|=s \cos \varphi \quad s_{2}=s \sin \varphi$ with $s>0$ and $\cos \varphi>0$ simplifies the distance factors which appear as arguments of the exponentials in (2.15) to give $s$ cosh $(\theta-i \varphi)$. An elementary contour deformation argument then shows that (3.2) is independent of $\varphi$; this motivates the definition of an operator $B_{s}$ on $L^{2}\left(R_{1}\right)$ given by

$$
\begin{aligned}
\left(B_{s} f\right)(x)= & \frac{i}{2 \pi} \int_{-\infty}^{\infty} d y \exp (-|s|(\cosh x+\cosh y) / 2) \\
& \cdot \tanh ((x-y) / 2) f(y) .
\end{aligned}
$$

Evidently if $s \neq 0$ then $B_{s}$ is self-adjoint; moreover it is of Hilbert-Schmidt class. Thus it has a set $\left\{\lambda_{j}\right\}$ of eigenvalues which accumulate, if anywhere, to zero, and for which

$$
\sum \lambda_{j}^{2}=\int_{-\infty}^{\infty}\left|B_{s}(x, y)\right|^{2} d x d y .
$$

The associated eigenvectors spab $L^{2}\left(R_{1}\right)$.

Equation (3.2) reduces to the form

$$
L_{2 n}=(-1)^{n} \operatorname{Tr} B_{s}^{2 n} \text {. }
$$

The first problem in taking the scaling limit is to show that the limit $\{t \rightarrow 0 \pm$, $\gamma(0)|r|$ constant $\}$ held fixed does indeed exist for functions $H$ and $K$. Making the transformation $\sinh \theta=\gamma(0)^{-1} \omega$ for each vertex label lends to a new self-adjoint operator $\hat{A}_{s}$ on $L^{2}(R)$

$$
\left(\hat{A}_{s} f\right)\left(\theta_{1}\right)=\frac{i}{2 \pi} \int_{-\infty}^{\infty} d \theta_{2} \hat{h}\left(\theta_{1}\right) \hat{h}\left(\theta_{2}\right) \hat{e}\left(\theta_{1}, \theta_{2}\right) f\left(\theta_{2}\right)
$$

where

$$
\hat{e}\left(\theta_{1}, \theta_{2}\right)=e\left(\gamma(0) \sinh \theta_{1}, \gamma(0) \sinh \theta_{2}\right)
$$


and

$$
\hat{h}(\theta \mid s)=\chi_{s_{t}}(\theta) \frac{\gamma(0) \cosh \theta}{\sinh (\gamma(0) \sinh \theta)} h(\gamma(0) \sinh \theta \mid \mathbf{s} / \gamma(0))
$$

In the above $\chi_{S_{t}}$ is the characteristic function for the set $S_{t}=[-\pi / \gamma(0), \pi / \gamma(0)]$ (note $\gamma(0) \sim|t|$ as $t \rightarrow 0 \pm$ ). In terms of this operator we have

$$
H(s / \gamma(0))=\left(\hat{h},\left(1+\hat{A}_{s}^{2}\right)^{-1} \hat{h}\right)
$$

with the usual $L^{2}(R)$ scalar product and

$$
K(s / \gamma(0))=\frac{1}{2} \operatorname{Tr} \log \left(1+\hat{A}_{s}^{2}\right) .
$$

The corrections to scaling can be estimated by noting that

$$
K(s \mid \gamma(0))-\operatorname{Tr} \log \left(1+B_{s}^{2}\right)=\operatorname{Tr} \log \left(1+\left(1+B_{s}^{2}\right)^{-1}\left(\hat{A}_{s}^{2}-B_{s}^{2}\right)\right)
$$

Provided that, for given $s_{0}>0,|s| \geqq s_{0}$ the estimate

$$
\begin{aligned}
& \left|K(\mathrm{~s} / \gamma(0))-\operatorname{Tr} \log \left(1+B_{\mathrm{s}}^{2}\right)\right| \\
& \quad \leqq\left(M\left(s_{0}\right) t^{2}+N\left(s_{0}\right) e^{-\pi s_{0} /|t|}\right) e^{-|s|}
\end{aligned}
$$

can be derived by tedious but elementary methods. The correction of $H$ may be estimated similarly.

Since both $\hat{m}$ and $m^{*}$ vanish as $|t|^{1 / 8}$ it is clear that $p=1 / 4$ should be chosen in (3.1). Let $F_{ \pm}(s)$ be defined by

$$
\begin{aligned}
F_{ \pm}(s)= & A^{ \pm}\left\{\left(\hat{h},\left(1+B_{s}^{2}\right)^{-1} \hat{h}\right),(\text { resp. } 1)\right\} \\
& \cdot\left\{\exp \left(\operatorname{Tr} \log \left(1+B_{s}^{2}\right)\right)\{+(0, \text { resp. }(-1))\}\right.
\end{aligned}
$$

for $\left(T>T_{C}, F_{+}\right)\left[\operatorname{resp} .\left(T<T_{C}, F_{-}\right)\right]$where $A^{ \pm}$are constants which may be determined. Then we have

Proposition 3.1. Provided $s \geqq s_{0}>0$,

$$
\lim _{t \rightarrow 0 \pm}|t|^{1 / 4} \varrho(s / \gamma(0))=F_{ \pm}(s)
$$

and, moreover,

$$
\left.|| t\right|^{1 / 4} u_{2}(s / \gamma(0))-\left.F_{ \pm}(s)\left|\leqq \alpha_{ \pm}\right| t\right|^{5 / 4} F_{ \pm}(s)+\beta\left(s_{0}\right)|t|^{9 / 4} e^{-|s|} .
$$

Remarks. The first term on the r.h.s. of (3.15) comes from the expansions of $\hat{m}$ and $m^{*}$ about $t=0$. The other term is the "correction to scaling" which arises from (3.12).

The scaled functions $F_{ \pm}(s)$ also have a representation of type (2.2) from which the following proposition may be derived:

Proposition 3.2. $F_{ \pm}(s)$ is represented by a series of positive terms, convergent for any $s>0$; each term is convex for $s \geqq s_{0}>0$ and so the sum is convex there and, moreover, continuous. 
Recall the integral representation of the zeroth-order Bessel function of the second kind:

$$
K_{0}(s)=\frac{1}{2 \pi} \int_{-\infty}^{\infty} e^{-|s| \cosh \theta} d \theta
$$

By elementary examination of the linked cluster result (3.13), using convexity of the $\log$ function, we have

Proposition 3.3. The scaling functions $F_{ \pm}(s)$ satisfy the estimates

$$
\begin{aligned}
& F_{+}(s) \leqq K_{0}(s) \exp \left(K_{0}(s)^{2}\right), \\
& F_{-}(s) \leqq \exp \left(K_{0}(s)^{2}\right)-1 .
\end{aligned}
$$

Remarks. Propositions 5.2 and 5.3 ensure integrability of $s^{n} F_{ \pm}(s)$ on any set $\left[s_{0}, \infty\right), s_{0}>0$.

\section{Duality}

By analogy with the translational operator $T$ for the spins themselves, operations may be defined directly for the spinors given by

$$
\Gamma_{2 j-1}=f_{j}^{\dagger}+f_{j}, \Gamma_{2 j}=-i\left(f_{j}^{\dagger}-f_{j}\right), 1 \leqq j \leqq M .
$$

One such operation is

$$
\begin{aligned}
& \mathscr{D} \Gamma_{j} \mathscr{D}^{\dagger}=\Gamma_{j-1}, \quad j=2, \ldots 2 M \\
& \mathscr{D} \Gamma_{1} \mathscr{D}^{\dagger}=-\Gamma_{2 M} .
\end{aligned}
$$

Under this transformation we have

$$
\begin{aligned}
& \mathscr{D} V_{1}(x) \mathscr{D}^{\dagger}=\exp i x\left(\sum_{1}^{M-1} \Gamma_{2 j} \Gamma_{2 j+1}-\Gamma_{2 M} \Gamma_{1}\right) \\
& \mathscr{D} V_{2}(x) \mathscr{D}^{\dagger}=\exp i x\left(\sum_{1}^{M-1} \Gamma_{2 j-1} \Gamma_{2 j}+P \Gamma_{2 M-1} \Gamma_{2 M}\right) .
\end{aligned}
$$

Consideration of the van Hove sequence $\{N \rightarrow \infty$, then $M \rightarrow \infty\}$ implies that only the term $V_{+}^{N-r} \sigma_{1}^{x} V_{-}^{r} \sigma_{1}^{x}$ need be considered in the 2-point correlation function expression. Under the duality transformation $V$ is replaced by $V^{\prime}$ but with the inclusion of misfit bonds between the points:

$$
\operatorname{Tr} V_{+}^{N-r} \sigma_{1}^{x} V_{-}^{r} \sigma_{1}^{x}=\operatorname{Tr}\left(V_{+}^{\prime}\right)^{N-r} \sigma_{1}^{x}\left(\hat{V}^{\prime}\right)^{r} \sigma_{1}^{x} .
$$

Thus $\hat{V}^{\prime}$ has a line of reversed $K_{2}$ bonds in the $V_{1}\left(K_{2}\right)$ factors between the points, which are removed by moving one of the spin operators $\sigma_{1}^{x}$ up to the other one. With this alternative operator picture we derive the relationship

$$
\mathscr{D}:\left\langle\sigma_{1} \sigma_{1+r}\right\rangle=Z_{t}(\Delta)^{-1} \operatorname{Tr}\left\{\left(V_{+}^{\prime}\right)^{N-r}\left(V^{\prime}\right)^{r}+\left(V^{\prime}\right)^{N-r}\left(V_{+}^{\prime}\right)^{r}\right\},
$$

where the r.h.s. has $K_{1}^{*}$ and $K_{2}$ interchanged. The reader may check that the $T<T_{C}$ and $T>T_{C}$ results in Sections 1 and 2 of this paper are correctly related by duality. 
(Note the parameter mapping $A \rightarrow A, B \rightarrow 1 / B$.) This provides a rationalisation of the interplay between Pfaffians and generalised Pfaffians in this problem. One should notice that (4.6) is precisely the same as the usual statement of duality [5]. The operation (4.2) enables one also to understand the relationship between surface tension and inverse correlation length [6] and the relationship between the transfer matrices for pure phases [7] with "all +" or "all - " boundary conditions and the single phase transfer matrix with free edges [8]. By taking the limit $N \rightarrow \infty$, then $r \rightarrow \infty$ in (4.6) we obtain the boundary condition $F(\emptyset)$ which is appropriate for $V^{1}$. Evidently

$$
\left\langle\Phi_{-}\left|\sigma_{1}^{x}\right| \Phi_{+}\right\rangle\left(K_{1}^{*}, K_{2}\right)=\left\langle\Phi_{-}^{\prime} \mid \Phi_{+}^{\prime}\right\rangle\left(K_{2}, K_{1}^{*}\right)
$$

from which it follows that

$$
\lim _{M \rightarrow \infty}\left\langle\Phi^{\prime} \mid \Phi_{+}^{\prime}\right\rangle\left(K_{2}, K_{1}^{*}\right)=m^{*}\left(K_{1}^{*}, K_{2}\right),
$$

where $m^{*}$ is the spontaneous magnetisation; this has been shown to be $[9,10]$

$$
m^{*}\left(K_{1}^{*}, K_{2}\right)=\left[1-\left(\sinh 2 K_{1}^{*} / \sinh 2 K_{2}\right)^{2}\right]^{1 / 8} .
$$

By using the symmetrised form $V^{1}$ in the expression for the pair correlation the relationship

$$
\begin{aligned}
\left\langle\Phi_{-}\right. & \left.\left|\sigma_{1}^{x}\right| \Phi_{+}\right\rangle \\
= & \cosh K_{1}^{*}\left\langle\Phi^{\prime}\left|\sigma_{1-}^{x}\right| \Phi_{+}^{\prime}\right\rangle \\
& -\sinh K_{1}^{*}\left\langle\Phi_{-}^{\prime}\left|\sigma_{1}^{y}\right| \Phi_{+}^{\prime}\right\rangle
\end{aligned}
$$

may be derived by taking the appropriate limits. The second matrix element on the right hand side of (4.10) is known to be zero [10]. The remaining results in Table 1 for the initial conditions can then be derived immediately (note that $\sinh 2 K_{1}^{*} \sinh 2 K_{1}=1$ ). This use of duality considerably facilitates the older derivation [11].

\section{Discussion}

In this paper, a new representation for the pair correlation function of the Rectangular Ising Ferromagnet has been derived using the techniques of Ref. [1]. In addition, the scaling limit has been proved to exist for non-zero scaling lengths; continuity and monotonicity have been established for the scaling function, and bounds have been placed on its behaviour, giving integrability results. Barouch, McCoy, Tracy, and $\mathrm{Wu}$ [12] have obtained similar expressions, but did not apparently have the operator formalism at their disposal. Thus the analytic results which they obtained were rather weak; but, of very considerable interest, they derived, and analysed, a differential equation for a function related to $H(s)$. The present results can probably make this derivation rigorous. But this still leaves the vexing question of the integrability $s F_{ \pm}(s)$ at $s=0$, and the validity of the scaling limit there. The adduction of duality in Section 4 will, it is to be hoped, add some coherence to a "troubled republic" of formulae.

Acknowledgements. The author wishes to thank J. Glimm, J. Groeneveld, T. Spencer, and A. Zepeda very much for useful conversations. He also acknowledges the support and hospitality of the Aspen Center for Physics, the I.H.E.S. and the Lab. de Physique Theorique at the E.P.F.-Lausanne. 


\section{References}

1. Abraham,D.B.: Commun. math. Phys. 59, 17 (1978)

2. Caianiello,E.R.: Combinations and renormalisation in quantum field theory. New York: Benjamin 1973

3. Kasteleyn,P.W.: Graph theory and theoretical physics (ed. F. Harary). New York : Academic Press 1967

4. Hove,L.van: Lectures on the many body problem (ed. E. R. Caianiello). New York: Academic Press 1962

5. Mittag,L., Stephen, M.J.: J. Math. Phys. 12, 441 (1971), and References therein

6. Abraham,D. B., Reed,P.: Phys. Rev. Letters 33, 377 (1974), Ref. [10] therein

7. Abraham,D.B., Martin-Löf,A.: Commun. math. Phys. 32, 245-268 (1973)

8. Abraham,D.B.: Studies Appl. Math. 50, 71 (1971)

9. Martin-Löf, A.: Commun. math. Phys. 24, 253 (1972); Lebowitz,J.L., Martin-Löf, A.: Commun. math. Phys. 25, 276 (1972); Bennettin,G., Gallavotti,G., Jona-Lasinio, G., Stella, A.L. : Commun. math. Phys. 30, 45 (1973); see also Ref. [7]

10. Yang, C. N.: Phys. Rev. 85, 808 (1952)

11. Abraham,D.B.: Studies Appl. Math. 51, 179 (1972)

12. Wu,T.T., McCoy,B.M., Tracy,C.A., Barouch,E.: Phys. Rev. B 13, 316 (1976); Tracy,C.A., McCoy, B.M.: Phys. Rev. B12, 368 (1975)

Communicated by E. Lieb

Received December 27, 1977 